\title{
Projection specificity in heterogeneous locus coeruleus cell populations: implications for learning and memory
}

\author{
Akira Uematsu, ${ }^{1}$ Bao Zhen Tan, ${ }^{1}$ and Joshua P. Johansen ${ }^{1,2}$ \\ ${ }^{1}$ RIKEN Brain Science Institute, Laboratory for Neural Circuitry of Memory, 2-1 Hirosawa, Wako-shi, Saitama 351-0198, Japan; \\ ${ }^{2}$ Department of Life Sciences, Graduate School of Arts and Sciences, University of Tokyo, Tokyo 153-8902, Japan
}

\begin{abstract}
Noradrenergic neurons in the locus coeruleus (LC) play a critical role in many functions including learning and memory. This relatively small population of cells sends widespread projections throughout the brain including to a number of regions such as the amygdala which is involved in emotional associative learning and the medial prefrontal cortex which is important for facilitating flexibility when learning rules change. LC noradrenergic cells participate in both of these functions, but it is not clear how this small population of neurons modulates these partially distinct processes. Here we review anatomical, behavioral, and electrophysiological studies to assess how LC noradrenergic neurons regulate these different aspects of learning and memory. Previous work has demonstrated that subpopulations of LC noradrenergic cells innervate specific brain regions suggesting heterogeneity of function in LC neurons. Furthermore, noradrenaline in mPFC and amygdala has distinct effects on emotional learning and cognitive flexibility. Finally, neural recording data show that LC neurons respond during associative learning and when previously learned task contingencies change. Together, these studies suggest a working model in which distinct and potentially opposing subsets of LC neurons modulate particular learning functions through restricted efferent connectivity with amygdala or mPFC. This type of model may provide a general framework for understanding other neuromodulatory systems, which also exhibit cell type heterogeneity and projection specificity.
\end{abstract}

Learning and memory is critical to our survival as it facilitates adaptive behavioral decision-making. Depending on the circumstances, different types of behavioral memories are formed and sometimes these memories require alteration to match a constantly changing environment. The process of forming and maintaining associative behavioral memories or flexibly altering behavioral strategies when task demands change recruits partially separable neural circuits in the amygdala and medial prefrontal cortex (mPFC), respectively (LeDoux 2000; Arnsten 2009). The amygdala is important for emotional memory formation in which sensory stimuli are associated with aversive (or rewarding) outcomes to enable adaptive behavioral responses (Davis and Whalen 2001; Johansen et al. 2011; Duvarci and Pare 2014; Herry and Johansen 2014; Janak and Tye 2015; Tovote et al. 2015). In contrast, the mPFC is involved in cognitive flexibility during learning, facilitating switches to new behavioral strategies to optimize adaptive behavior (Arnsten 2009, 2011).

Noradrenaline neurons in the locus coeruleus (LC) have been implicated in both emotional associative memory formation as well as cognitive flexibility during learning (Berridge and Waterhouse 2003; Aston-Jones and Cohen 2005; Arnsten 2009; Sara and Bouret 2012). One hypothesis that has been proposed is that noradrenaline action in amygdala engages more reflexive adaptive behaviors while noradrenaline in mPFC facilitates cognitive flexibility (Arnsten 2009). How LC noradrenaline neurons regulate these different aspects of learning and memory is an important open question. A commonly held view is that a homogenous population of LC neurons provides a common input to all LC efferent targets including the amygdala and mPFC. According to this view, the specificity of this homogeneous noradrenaline signal would be controlled through its interaction with functionally distinct brain regions. Another possibility is that partially dis-

Corresponding author: jjohans@brain.riken.jp

Article is online at http://www.learnmem.org/cgi/doi/10.1101/Im.037283.114. tinct populations of LC neurons project to the amygdala and $\mathrm{mPFC}$ and that these heterogeneous LC cell populations directly facilitate emotional learning or cognitive flexibility. This latter scenario in which different populations of cells are defined, at least partially, by their distinct efferent connectivity can be termed projection or efferent specificity. Projection specificity is apparent in Drosophila neuromodulatory networks where a number of studies have reported a high degree of connectional and functional specificity in distinct populations of neuromodulatory neurons (Liu et al. 2012; Waddell 2013). There is also evidence for projection specificity in mammalian dopamine neurons in the ventral tegmental area (Fallon 1981; Swanson 1982; Lammel et al. 2014; Fields and Margolis 2015). To understand whether LC-noradrenaline neurons exhibit projection specificity and whether this has functional consequences for learning and memory we will first review anatomical, brain manipulation and neural processing studies of LC. We will then integrate this information into a hypothetical model of LC function during learning and memory. We suggest that distinct, heterogeneous pools of LC neurons, based on their efferent connectivity, engage specific memory circuits depending on task requirements. This builds on previous work (Chandler et al. 2014a,b), but examines this idea in the context of functional neural circuits involved in specific learned behaviors. The ultimate goal of this review is to assimilate important information on LC function related to specific aspects of learning and memory and to help generate hypotheses and ideas for future study. We will focus on rodent and primate work as studies in these species have provided a wealth of information on noradrenaline circuits during learning and memory. For

\footnotetext{
(C) 2015 Uematsu et al. This article is distributed exclusively by Cold Spring Harbor Laboratory Press for the first 12 months after the full-issue publication date (see http://learnmem.cshlp.org/site/misc/terms.xhtml). After 12 months, it is available under a Creative Commons License (AttributionNonCommercial 4.0 International), as described at http://creativecommons. org/licenses/by-nc/4.0/.
} 
excellent reviews on more general aspects of LC function see Berridge and Waterhouse (2003), Aston-Jones and Cohen (2005), and Sara and Bouret (2012).

\section{Anatomical connectivity and efferent specificity in LC neurons}

The LC consists of a small number of NA containing neurons $(\sim 1500$ in the rat, 15,000 in the human/hemisphere), which project widely throughout the brain and receive inputs from a diverse array of brain regions. From the brainstem and midbrain, LC neurons receive input from the reticular formation, nucleus tractus solitarius, vestibular nucleus, nuclei gigantocellularis and paragigantocellularis, and the periaqueductal gray conveying information about visceral and sympathetic nervous system function as well as pain and threat (Cedarbaum and Aghajanian 1978; Aston-Jones et al. 1986; Van Bockstaele et al. 1998a). Forebrain structures including the dorsomedial, lateral, and paraventricular nuclei of the hypothalamus, central nucleus of the amygdala, bed nucleus of the stria terminalis, insular cortex, and prefrontal cortex provide complex emotional, homeostatic, and cognitive information to LC neurons (Cedarbaum and Aghajanian 1978; Arnsten and Goldman-Rakic 1984; Luppi et al. 1995; Van Bockstaele et al. 1998b; Reyes et al. 2005). The LC is also interconnected with various neuromodulatory brain regions including the ventral tegmental area (dopamine) and dorsal raphe (serotonin) (Palkovits et al. 1977; Swanson 1982; Deutch et al. 1986; Ornstein et al. 1987). Together, these afferent connections allow for modulation of LC neural processing by basic sensory and visceral experiences as well as regulation by top-down influences from forebrain structures conveying highly processed cognitive/emotional information (Fig. $1 \mathrm{~A}$, see Berridge and Waterhouse 2003; Aston-Jones and Cohen 2005; Sara and Bouret 2012) for detailed anatomical citations of this work).

Despite the small number of neurons in the LC, it projects broadly to most forebrain regions as well as some midbrain and brainstem nuclei and the cerebellum and spinal cord (for review, see Berridge and Waterhouse 2003; Aston-Jones and Cohen 2005; Sara and Bouret 2012; Valentino and Van Bockstaele 2015). Related to learning and memory, the LC sends strong efferent projections to the amygdala (lateral, basal, and central nuclei) and mPFC (Fallon et al. 1978; Arnsten 2009). Although the LC has traditionally been viewed as a homogenous population of cells (Fig. 1B), anatomical studies have demonstrated some specificity in the projections of LC neurons. This suggests a degree of anatomical (and possibly functional) hetero- geneity. As an example of this, early studies using single retrograde tracer injections into different brain regions found some limited topographical organization of LC efferents, with nonoverlapping subregions of the LC projecting to distinct efferent targets (Mason and Fibiger 1979; Waterhouse et al. 1983, 1993; Loughlin et al. 1986). However, using this single retrograde tracer approach, it is apparent that much of the LC contains cells, which project to multiple brain regions. Thus, if there were cell heterogeneity based on efferent targeting it would arise from intermixed populations of LC neurons. To adequately determine whether individual cells in an intermixed population project to specific brain 
regions, a combinatorial strategy using two or more retrograde tracers is necessary. Using this type of approach, several studies found a high degree of heterogeneity in LC neurons with respect to their efferent connectivity. One study injected three different fluorescent retrograde tracers into the orbitofrontal cortex (OFC), mPFC, and anterior cingulate cortex (ACC) (Chandler and Waterhouse 2012). Interestingly, largely nonoverlapping cell populations projecting to these three regions were detected in the LC. A related follow-up study found that these unique subpopulations of LC neurons projecting to OFC, mPFC, and ACC were also distinct from another population of LC neurons projecting to motor cortex (M1) (Fig. 1C; Chandler et al. 2014a). Importantly, OFC and mPFC projecting LC neurons displayed a different molecular profile from M1 neurons, expressing higher transcript levels of proteins associated with glutamatergic transmission and excitability. Moreover, in slice preparation studies these cells were found to be more excitable and have higher baseline firing rates compared with M1 neurons. This along with prior studies demonstrating that subpopulations of LC noradrenaline neurons coexpress different neurotransmitters (Berridge and Waterhouse 2003) suggests that distinct classes of LC neurons exhibit unique molecular identities along with projection specificity.

Although the anatomical studies reveal that distinct LC neurons project to specific brain regions, other studies using a variety of anatomical approaches have found that LC neurons are homogenous and exhibit more collateralization in their efferent connectivity (Nakamura and Iwama 1975; Nagai et al. 1981; Room et al. 1981; Schwarz et al. 2015). It will be important in future work to determine the degree of collateralization and specificity individual populations of LC neurons exhibit in their efferent connectivity. For example, the structures innervated by a given subpopulation of LC cells may be governed by some functional demand. Consistent with this, using a double retrograde tracer approach one study found that subpopulations of LC neurons send axon collaterals to the somatosensory thalamus and cortex, but less to visual cortical/thalamic brain regions (Simpson et al. 1997). This suggests that functional demands may underlie the connectivity and the degree of collateralization that individual populations of LC neurons exhibit. Relating this to learning and memory, it is possible that distinct classes of LC-noradrenaline cells projecting to functionally distinct memory networks such as the amygdala or mPFC (and functionally related regions) may modulate specific forms of learning and memory.

\section{LC noradrenaline neurons participate in emotional associative learning and cognitive flexibility}

While anatomical studies have revealed broad projections of LC noradrenaline neurons and demonstrated some degree of projection specificity, the functional studies of LC have established the causal importance of this system in various forms and aspects of behavioral learning and memory. Many studies using neurotoxic or electrolytic lesions of the LC or of the dorsal noradrenergic bundle (a fiber bundle containing LC noradrenaline axons targeted to specific forebrain targets) or LC specific pharmacological manipulations have found effects on various aspects of learning and memory including fear learning, extinction and reversal learning, avoidance, and working memory (Mason and Iversen 1975; Fibiger and Mason 1978; Plaznik and Kostowski 1980; Cole and Robbins 1987; Tsaltas et al. 1989; Selden et al. 1990; Harris and Fitzgerald 1991; Langlais et al. 1993; Neophytou et al. 2001; Sears et al. 2013; Soya et al. 2013).

Although these studies suggest that LC neurons are important in many different types of learning and memory one impor- tant caveat is that some of the key findings have not been replicated by other labs (Amaral and Foss 1975; Fibiger and Mason 1978; Koob et al. 1978; Tsaltas et al. 1984, 1989; Selden et al. 1990). It is possible that lesion technique, differences in behavioral paradigms and/or compensation from spared noradrenaline fibers, or receptor systems or even other neuromodulatory networks could have contributed to the variability in the findings. Another possibility is that global manipulations of many functionally distinct, possibly competing, LC neuronal subpopulations could have produced variable or null effects on the behavior. More precise anatomical, genetic and/or temporal manipulations may help to resolve these disparities in the literature and offer important insights into LC function.

One way to more precisely study the involvement of LCnoradrenaline in distinct aspects of learning and memory is to manipulate adrenergic receptor signaling or LC fibers in specific brain regions. Using this approach the role of noradrenaline in the lateral and basal nuclei of the amygdala (LA/B) has been examined. The LA/B is an important site of plasticity through which sensory stimuli (auditory, visual, etc.) become associated with aversive or rewarding outcomes to allow them access to behavioral and visceral circuits involved in producing defensive or reward-seeking behaviors (LeDoux 2000; Davis and Whalen 2001; Johansen et al. 2011; Duvarci and Pare 2014; Herry and Johansen 2014; Janak and Tye 2015; Tovote et al. 2015). The aversive form of this learning has been termed fear conditioning and noradrenaline in the LA/B is particularly important for the acquisition of fear memories. For example, injections of $\beta$-adrenergic $(\beta-\mathrm{AR})$ receptor antagonists into the $\mathrm{LA} / \mathrm{B}$ reduce the acquisition of fear learning (Fig. 2A; Bush et al. 2010). In contrast, intra-LA/ $\beta$ antagonists given immediately following learning or before a memory expression test have no effect on behavior. This suggests that $\beta$-AR activation in this region is important during fear learning, but not necessary for consolidation or expression of fear memories. Aversive footshock produces phasic activation of LC neurons and increases in noradrenaline levels in the amygdala (Galvez et al. 1996; Quirarte et al. 1998) which in turn modulates the firing rate of LA/B neurons (Buffalari and Grace 2007; Chen and Sara 2007). This suggests that phasic, footshock evoked activation of $\beta$-ARs on LA/B neurons could modulate fear learning. These effects of noradrenaline in LA/B occur through noradrenergic modulation of Hebbian plasticity mechanisms (Johansen et al. 2014) possibly by reducing feedforward inhibition and/or through $\beta$-AR mediated modulation of calcium-dependent signaling processes (Tully et al. 2007; Johansen et al. 2011). Once a fear memory has been consolidated, recall of that memory places it into a labile state (a process termed reconsolidation) where it can be changed or disrupted through manipulation of specific signaling pathways in LA neurons (Nader and Hardt 2009). In addition to amygdala noradrenaline involvement in fear memory formation, intra-LA/B $\beta$-AR blockade abolishes and stimulation enhances fear memory reconsolidation (Debiec and Ledoux 2004; Debiec et al. 2011). In addition to its role in directly regulating plasticity mechanisms in the LA/B mediating fear learning, adrenergic receptor activation in LA/B is important in modulating hippocampal-dependent memories (McGaugh 2004; Berlau and McGaugh 2006; Fiorenza et al. 2012). Overall, what is clear is that noradrenaline in the LA/B is important for fear memory formation and reconsolidation while also playing a role in modulating other forms of learning during the memory consolidation period. It will be imperative in future work to determine whether the LC is the functional source of noradrenaline to the amygdala and how amygdala projecting LC neurons encode information during fear learning and reconsolidation. It will also be important to examine whether noradrenaline in the amygdala modulates appetitive learning. 
A

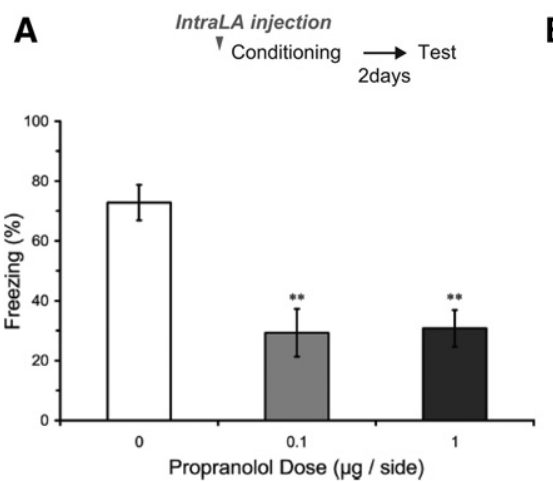

B
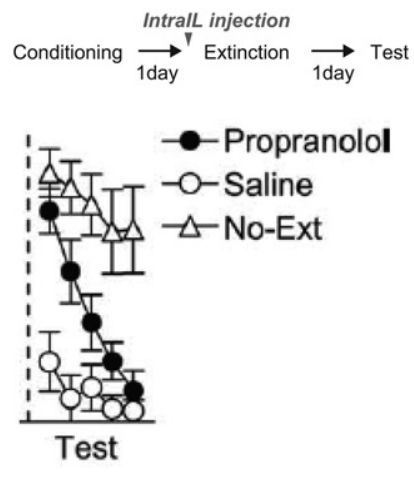

Figure 2. Examples of specific effects of noradrenaline manipulations in amygdala or medial prefrontal cortex (mPFC). (A) $\beta$-Adrenergic receptor ( $\beta$-AR) blockade in amygdala reduces fear memory formation. When auditory cues are paired with aversive footshocks during training freezing responses develop to the auditory cues providing a measure of fear. Intralateral amygdala (LA) injection of a $\beta$-AR antagonist (two different doses, 0.1 and $1.0 \mu \mathrm{g} /$ side, $x$-axis) before fear conditioning reduces memory formation (freezing, $y$-axis) measured at "Test" $48 \mathrm{~h}$ later (adapted from Bush et al. 2010). (B) $\beta$-AR blockade in the infralimbic (IL) portion of the MPFC reduces extinction memory consolidation. Following fear learning, repeated presentation of the auditory cue results in reduction of fear/freezing responses (termed extinction learning). Intra IL injections of a $\beta$-AR antagonist before extinction learning reduces extinction memory consolidation as evidenced by higher freezing levels ( $y$-axis) upon cue presentation $24 \mathrm{~h}$ after extinction training in the antagonist (propranolol) treated compared with the vehicle (saline) treated group (adapted from Mueller et al. 2008).

Although the amygdala and mPFC interact through reciprocal connectivity and some of their behavioral functions overlap (Sotres-Bayon and Quirk 2010; Likhtik et al. 2014; Senn et al. 2014), the mPFC and noradrenaline in this region is thought to be more important for behavioral flexibility, strategic planning, and working memory (Arnsten 2009, 2011). For example, in contrast to its role in the acquisition of fear learning in the amygdala, $\beta$-AR and $\alpha 1$-adrenergic receptor ( $\alpha 1$-AR) activation in the infralimbic region of the mPFC is necessary for reversing fear and reward-related behavioral memories when they are no longer appropriate (see Fig. 2B for an example of these findings), a process called extinction learning (Mueller et al. 2008; Do-Monte et al. 2010; LaLumiere et al. 2010). Supporting a role for mPFC noradrenaline in regulating behavioral flexibility, noradrenaline levels in the mPFC are increased during fear extinction training (Feenstra et al. 2001; Hugues et al. 2007). In addition, $\alpha 2$-adrenergic receptors ( $\alpha 2$-ARs) in the prelimbic region of the mPFC are involved in working memory and reversal learning. Specifically, $\alpha 2$-AR receptor activation in the prelimbic cortex is necessary for optimal performance on a working memory version of the T-maze task and readjustments of behavioral strategy following errors (Caetano et al. 2012). Furthermore, noradrenaline denervation of the mPFC results in reductions in reversal learning when animals are faced with changes in task structure (McGaughy et al. 2008; Newman et al. 2008). These types of cognitive deficits are also evident in monkeys (for review, see Arnsten 2009). The apparent deficits in reversal learning following manipulations of noradrenaline in $\mathrm{MPFC}$ are consistent with theoretical ideas of the role of noradrenaline in signaling "unexpected uncertainty" (Yu and Dayan 2005) which occurs with contingency reversals. Increases in tonic and reductions in phasic, task-related firing rates in LC noradrenaline neurons has been suggested to favor exploratory, as opposed to task directed, behaviors to facilitate the discovery of new optimal learning strategies (Aston-Jones and Cohen 2005). This exploratory type of behavior could occur following contingency changes. Related to this, stimulation of LC noradrenergic fibers in the mPFC during a complex decisionmaking task produces stochastic/exploratory behavior when goaldirected decision-making is optimal (Tervo et al. 2014). In contively. However, based on this data alone

trast, in animals that have been trained to exhibit constant stochastic/exploratory behavior, inhibiting LC terminals in the mPFC produces a switch to an optimal, goal-directed decision-making strategy. Together, the available data suggest that noradrenaline in the MPFC is important in behavioral flexibility including extinction and reversal learning. Dynamic regulation of tonic and phasic noradrenaline release in mPFC could facilitate behavioral flexibility and switches to new, optimal behavioral strategies.

These studies on the role of LC and noradrenaline in the amygdala and mPFC demonstrate that noradrenaline has distinct effects on specific aspects of learning and memory depending on the brain region it modulates. Based on this and the fact that some LC-noradrenaline cells have distinct connectivity with their efferent targets it is possible that different subsets of LC neurons projecting to amygdala or mPFC modulate either the formation of emotional associative memories or cognitive flexibility, respecit is also possible that the divergent effects of noradrenaline on these different brain regions are governed by local processes within the amygdala or MPFC and not by unique populations of LC neurons. To properly address this question, modern anatomical and cell type-specific manipulations including cell type-targeted anatomical tracing approaches as well as opto- or chemogenetic manipulations of anatomically defined neuronal populations (Luo et al. 2008; Johansen et al. 2012; Tye and Deisseroth 2012) are necessary. This would allow a determination of whether distinct LC cell populations project to amygdala and mPFC and whether these cells are functionally dissociable.

\section{Neural coding in LC neurons}

The evidence for projection specificity and the differences in the effects of noradrenaline manipulations in mPFC and amygdala suggests that different populations of LC neurons may encode information in distinct ways depending on the brain regions they innervate. This implies that LC neural coding should be heterogeneous in some way and not uniform across the population of LC noradrenaline neurons. While technical limitations have made it difficult to measure neural activity from LC cells that project to specific brain regions, many studies have examined the firing properties of LC neurons in-vivo to elucidate their responsivity to basic sensory events and understand how learning alters these neural representations.

Noradrenaline neurons in LC have traditionally been characterized as having low baseline firing rates $(\sim 1-3 \mathrm{~Hz})$ which is modulated by wakefulness (see Berridge and Waterhouse 2003; Aston-Jones and Cohen 2005; Sara and Bouret 2012 for reviews of basic response properties of LC neurons). In addition, LC cells are multimodal and respond to many different types of sensory and visceral stimuli including aversive and rewarding outcomes. The initial responses to sensory and visceral stimuli appear to be somewhat uniform across all LC neurons suggesting homogeneity in processing these types of experiences. Interestingly, these sensory and visceral-related responses are context dependent and strongly regulated by learning and task performance. One 
example of this is that with repeated experience, LC neural responses to a variety of sensory stimuli are reduced, a process termed habituation (Aston-Jones and Bloom 1981; Sara and Segal 1991; Herve-Minvielle and Sara 1995). Importantly, habituation responses are not uniform across all LC cells (Sara and Segal 1991) suggesting that heterogeneity in neural coding can emerge with experience during simple forms of learning. Later studies examined the firing properties of LC neurons during more complex learning and memory tasks in which sensory cues or a combination of sensory stimuli and behavioral responses predicted aversive or rewarding outcomes. Generally, these studies found that LC neurons responded more to sensory cues predicting reward or punishment (Rasmussen and Jacobs 1986; Sara and Segal 1991; Aston-Jones et al. 1994, 1997; Usher et al. 1999; Bouret and Sara 2004; Rajkowski et al. 2004; Bouret and Richmond 2009). In some of these studies, a behavioral response was required following the sensory cue to achieve reward. Under these circumstances, the sensory stimulus elicited modulation of LC neural firing rate became better time-locked to the behavioral response than to the sensory cue itself (Bouret and Sara 2004; Rajkowski et al. 2004; Bouret and Richmond 2009). However, this was not a purely behavior elicited change in firing rate as it was not apparent when animals produced the same behavior in the absence of the predictive cue (Bouret and Richmond 2009). Importantly, while some studies reported homogeneity in the response of LC neurons during these types of learning tasks, other work suggested that distinct subsets of LC neurons encode reward predictive sensory cues, task-related behavioral responses or both (Bouret and Richmond 2009, 2015; Kalwani et al. 2014).

During contingency reversals or extinction, when task contingencies change, the baseline or tonic firing rate of LC neurons increases and the phasic, sensory cue elicited responses in LC neurons eventually changes to reflect the new cue-outcome contingencies (Sara and Segal 1991; Aston-Jones et al. 1997; Usher et al. 1999). This increase in the tonic firing rate of LC neurons is also evident when animals are performing poorly on a task. Under these circumstances, this change in tonic firing rate is accompanied by a loss of phasic, task-related (sensory cue elicited for example) responding (Usher et al. 1999) (but see Kalwani et al. 2014). This suggests that during periods when animals are either focused on other variables in the environment or when they need to change their behavioral strategy, the firing mode of LC neurons changes from sensory elicited, phasic firing mode to heightened tonic activity. As discussed above, the change in tonic and phasic firing modes of LC neurons has been proposed to facilitate exploratory or goal-directed behavior, respectively. Dynamic changes in these firing modes could also facilitate switching behavioral strategies during reversal or extinction learning.

In summary, LC neurons respond to a variety of sensory and visceral stimuli and their response properties are modulated during learning and memory tasks. Although it appears that LC cells respond homogeneously to primary sensory and visceral experiences, there is evidence for heterogeneity in LC neural responding during learning that may reflect differential top-down control of LC function. This could be implemented differentially in LC neurons projecting to amygdala or mPFC. It will be critical in future work to examine the neural coding properties of distinct subpopulations of LC neurons during different learning and memory tasks.

\section{A hypothetical model of LC function during learning and memory}

Based on the evidence presented above including previous anatomical/physiological studies showing projection specificity in the LC system (Chandler and Waterhouse 2012; Chandler et al. 2013, 2014a) we propose a conceptual, hypothetical model of LC function in which distinct subpopulations of LC noradrenergic neurons modulate specific aspects of learning and memory based on their projection specificity. Specifically, we propose that anatomically distinct populations of LC neurons project to either the amygdala or mPFC (Fig. 3). Since noradrenaline in the amygdala is involved in learning cue-aversive outcome associations and reconsolidating reactivated fear memories, we hypothesize that amygdala projecting LC neurons are activated by aversive outcomes and/or sensory predictive cues and that neural activity during those time periods is important for the learning and reconsolidation of emotional memories. In contrast, $\mathrm{mPFC}$ projecting LC neurons may respond more during contingency changes/reversals or extinction of cue/behavior-outcome contingencies as noradrenaline in the MPFC appears to be important for flexibility in learning under these conditions. In line with this we propose that changes in sensory cue evoked or tonic neural activity in mPFC projecting LC neurons is important in regulating changes in behavior during reversal learning and extinction. LC neural subpopulations may function independently of one another, with amygdala projecting cells being recruited solely during emotional associative learning and mPFC projecting cells being engaged when contingencies change or behavioral flexibility is required. However, emotional learning and changes or reversals in learning are in many instances opposing processes. As a result, an alternate possibility is that these distinct LC neural populations may function in parallel, but antagonistically during these different forms of learning. Related to this, it is possible that differential coding across LC neuronal subpopulations does not occur

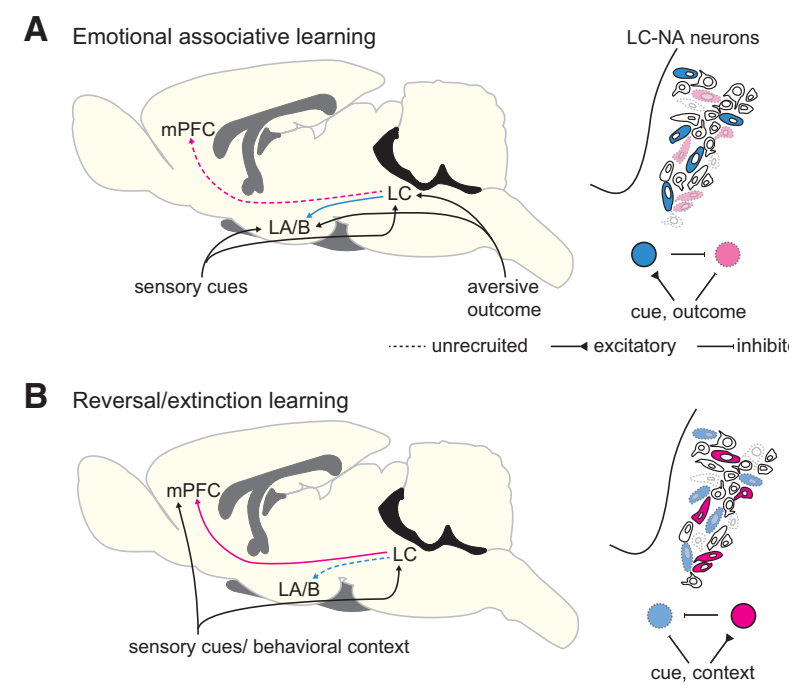

Figure 3. Hypothetical projection specificity model of locus coeruleus (LC) function during emotional associative learning (top) and reversal/extinction learning (bottom). On left, sagittal section of rat brain showing medial prefrontal cortex (mPFC, pink) and amygdala (blue) projecting LC neurons (adapted from Paxinos and Watson 1982). Insets on right show coronal mockup of LC with mPFC (pink) and amygdala (blue) projecting cells corresponding to the blue and pink lines in the sagittal sections. Below this are the hypothesized extrinsic and intrinsic functional connectivity (also depicted in the sagittal sections as inputs to LC), which could modulate interactions between these cell populations. Cells with dulled colors and dotted lines are those that are not engaged or recruited during the specific behavioral paradigm. Note that "behavioral context" and "context" refer to the learning context or state the animal is in (examples include alterations in contingency, task focus, etc.) which may or may not overlap with the physical environment. 
through completely distinct coding strategies, but rather through dynamic and more subtle shifts in the balance of task-related activity across individual LC cell populations.

A potential advantage of projection specificity in the LC is that it could allow for individual populations of LC neurons to control broadly distributed efferent target circuits subserving a distinct function (as suggested for LC innervation of somatosensory versus visual system (Simpson et al. 1997)). For example, a subpopulation of LC noradrenergic neurons may project to the amygdala, but also send collaterals to other functionally related brain regions to help coordinate activity across a distributed network involved in forming or reforming emotional associative memories. Another potential benefit to this type of anatomical arrangement could be to facilitate local interactions between functionally complementary or antagonistic neural subpopulations. This could occur through local network interactions between distinct cell populations in LC and/or through long range feedback connections from brain regions which receive input from the LC (Fig. 3). These interactions could provide an on-off switch for context dependent, dynamic regulation of functionally/anatomically distinct cell modules. Supporting these ideas, local connectivity as well as gap junction coupling between LC neurons has been documented and many brain regions which receive LC noradrenaline innervation send direct or indirect projections back to LC (Aghajanian et al. 1977; Egan et al. 1983; Ennis and Aston-Jones 1986; Christie et al. 1989; Christie and Jelinek 1993; Travagli et al. 1995; Ishimatsu and Williams 1996; Alvarez et al. 2002). To examine this hypothesis, future studies should determine how distinct LC neuronal populations are interconnected locally and through long range connectivity. This would then allow a determination of the functional importance of these interconnections for neural processing in distinct LC neuronal modules and behavior during learning and memory tasks.

Despite evidence for this model and for the importance of projection specificity in the LC noradrenaline system, there are still many open questions. For example, it hinges on the idea that different subpopulations of LC neurons project to amygdala and $\mathrm{mPFC}$ and that they serve distinct functions during different types of learning. However, there are many studies which report highly collateralized projection patterns of LC neurons (Nakamura and Iwama 1975; Nagai et al. 1981; Room et al. 1981; Schwarz et al. 2015) and it is not clear whether specific subpopulations of cells project to these regions or even, more generally, whether distinct behaviorally functional subclasses of LC neurons exist. Furthermore, this model may be too general. For example, the $\mathrm{mPFC}$ is composed of functionally independent subregions (infralimbic, prelimbic, and pregenual anterior cingulate cortices) and it is possible that distinct populations of LC noradrenaline neurons project to these different subregions. Even if there are distinct anatomically/genetically defined cell classes within LC, it is possible that they do not perform unique neural processing functions in the adult animal. For example, efferent-specific LC neuronal populations may function to guide distinct developmental processes occurring in different LC projection target regions, but operate homogeneously after development. A final alternate possibility is that projection specific cell populations may receive the same inputs and function identically with respect to their spiking output, but might co-express and deliver distinct neurotransmitters to their target structures.

To adequately test this model will require a multilevel approach utilizing cutting edge anatomical, neuronal recording, optogenetic, and behavioral techniques. This type of experimental approach could be used to determine whether specific anatomically and/or genetically defined populations of LC neurons project to the amygdala and $\mathrm{mPFC}$ and participate differentially in different aspects of learning and memory. Testing the function- al role of the different cell populations for behavior will be the most important goal as this can guide experimental interpretation and design in anatomical and cell recording studies. If distinct LC cell populations are differentially involved in behavior then it will be important to examine whether LC cell subpopulations receive distinct afferent inputs from different brain regions and/ or cell types within those regions. As discussed above, it will also be important to catalog the broad efferent connectivity of individual LC cell populations. This is because unique connectivity patterns in subpopulations of LC neurons may regulate distributed, but functionally related, brain circuits. It will also be essential to determine how anatomically or genetically identified populations of LC neurons encode information during learning and memory tasks. Studies such as these could reveal unique neuronal coding strategies in individual LC cell populations.

This working model and technical approach offers a potentially important framework for studying other aspects of LC function or even other neuromodulatory systems. LC noradrenaline neurons innervate most of the brain and participate in many functions outside of learning and memory. Understanding the anatomical and functional heterogeneity of LC neurons and how these cells interact during different experiences could help to define a unitary function of this important neuromodulatory system. It appears that other neuromodulatory circuits such as tegmental dopamine neurons as well as other nonmodulatory brain regions also exhibit projection specificity. Understanding projection specificity in the LC could provide important insights into what may be a general anatomical and functional organizational theme across neuromodulatory and other brain regions.

\section{Acknowledgments}

We thank Thomas McHugh and Tamas Madarasz for helpful comments on the manuscript. This work was supported by grants to J.P.J. from the Strategic Research Program for Brain Sciences from the Ministry of Education, Culture, Sports, Science and Technology (11041047) and Grants-in-Aid for Scientific Research on Innovative areas and Kiban B $(25710003,25116531)$, a grant to A.U. from Grants-in-Aid for Young Scientists B (26750380), a RIKEN Special Postdoctoral Researcher Fellowship (SPDR) A.U. and a RIKEN Foreign Postdoctoral Fellowship (FPR, 55800) to B.Z.T.

\section{References}

Aghajanian GK, Cedarbaum JM, Wang RY. 1977. Evidence for norepinephrine-mediated collateral inhibition of locus coeruleus neurons. Brain Res 136: 570-577.

Alvarez VA, Chow CC, Van Bockstaele EJ, Williams JT. 2002. Frequency-dependent synchrony in locus ceruleus: role of electrotonic coupling. Proc Natl Acad Sci 99: 4032-4036.

Amaral DG, Foss JA. 1975. Locus coeruleus lesions and learning. Science 188: $377-378$.

Arnsten AF. 2009. Stress signalling pathways that impair prefrontal cortex structure and function. Nat Rev Neurosci 10: 410-422.

Arnsten AF. 2011. Catecholamine influences on dorsolateral prefrontal cortical networks. Biol Psychiatry 69: e89-e99.

Arnsten AF, Goldman-Rakic PS. 1984. Selective prefrontal cortical projections to the region of the locus coeruleus and raphe nuclei in the rhesus monkey. Brain Res 306: 9-18.

Aston-Jones G, Bloom FE. 1981. Norepinephrine-containing locus coeruleus neurons in behaving rats exhibit pronounced responses to non-noxious environmental stimuli. J Neurosci 1: 887-900.

Aston-Jones G, Cohen JD. 2005. An integrative theory of locus coeruleus-norepinephrine function: adaptive gain and optimal performance. Annu Rev Neurosci 28: 403-450.

Aston-Jones G, Ennis M, Pieribone VA, Nickell WT, Shipley MT. 1986. The brain nucleus locus coeruleus: restricted afferent control of a broad efferent network. Science 234: 734-737.

Aston-Jones G, Rajkowski J, Kubiak P, Alexinsky T. 1994. Locus coeruleus neurons in monkey are selectively activated by attended cues in a vigilance task. J Neurosci 14: 4467-4480. 
Aston-Jones G, Rajkowski J, Kubiak P. 1997. Conditioned responses of monkey locus coeruleus neurons anticipate acquisition of discriminative behavior in a vigilance task. Neuroscience 80: 697-715.

Berlau DJ, McGaugh JL. 2006. Enhancement of extinction memory consolidation: the role of the noradrenergic and GABAergic systems within the basolateral amygdala. Neurobiol Learn Mem 86: 123-132.

Berridge CW, Waterhouse BD. 2003. The locus coeruleus-noradrenergic system: modulation of behavioral state and state-dependent cognitive processes. Brain Res Brain Res Rev 42: 33-84.

Bouret S, Richmond BJ. 2009. Relation of locus coeruleus neurons in monkeys to Pavlovian and operant behaviors. J Neurophysiol 101: 898-911.

Bouret S, Richmond BJ. 2015. Sensitivity of locus ceruleus neurons to reward value for goal-directed actions. J Neurosci 35: 4005-4014.

Bouret S, Sara SJ. 2004. Reward expectation, orientation of attention and locus coeruleus-medial frontal cortex interplay during learning. Eur $J$ Neurosci 20: 791-802.

Buffalari DM, Grace AA. 2007. Noradrenergic modulation of basolateral amygdala neuronal activity: opposing influences of $\alpha-2$ and $\beta$ receptor activation. J Neurosci 27: 12358-12366.

Bush DE, Caparosa EM, Gekker A, LeDoux JE. 2010. $\beta$-Adrenergic receptors in the lateral nucleus of the amygdala contribute to the acquisition but not the consolidation of auditory fear conditioning. Front Behav Neurosci 4: 154.

Caetano MS, Jin LE, Harenberg L, Stachenfeld KL, Arnsten AF, Laubach M. 2012. Noradrenergic control of error perseveration in medial prefrontal cortex. Front Integr Neurosci 6: 125.

Cedarbaum JM, Aghajanian GK. 1978. Afferent projections to the rat locus coeruleus as determined by a retrograde tracing technique. J Comp Neurol 178: 1-16.

Chandler D, Waterhouse BD. 2012. Evidence for broad versus segregated projections from cholinergic and noradrenergic nuclei to functionally and anatomically discrete subregions of prefrontal cortex. Front Behav Neurosci 6: 20.

Chandler DJ, Lamperski CS, Waterhouse BD. 2013. Identification and distribution of projections from monoaminergic and cholinergic nuclei to functionally differentiated subregions of prefrontal cortex. Brain Res 1522: 38-58.

Chandler DJ, Gao WJ, Waterhouse BD. 2014a. Heterogeneous organization of the locus coeruleus projections to prefrontal and motor cortices. Proc Natl Acad Sci 111: 6816-6821.

Chandler DJ, Waterhouse BD, Gao WJ. 2014b. New perspectives on catecholaminergic regulation of executive circuits: evidence for independent modulation of prefrontal functions by midbrain dopaminergic and noradrenergic neurons. Front Neural Circuits 8: 53.

Chen FJ, Sara SJ. 2007. Locus coeruleus activation by foot shock or electrical stimulation inhibits amygdala neurons. Neuroscience 144: 472-481.

Christie MJ, Jelinek HF. 1993. Dye-coupling among neurons of the rat locus coeruleus during postnatal development. Neuroscience 56: 129-137.

Christie MJ, Williams JT, North RA. 1989. Electrical coupling synchronizes subthreshold activity in locus coeruleus neurons in vitro from neonatal rats. J Neurosci 9: 3584-3589.

Cole BJ, Robbins TW. 1987. Dissociable effects of lesions to the dorsal or ventral noradrenergic bundle on the acquisition, performance, and extinction of aversive conditioning. Behav Neurosci 101: 476-488.

Davis M, Whalen PJ. 2001. The amygdala: vigilance and emotion. Mol Psychiatry 6: 13-34.

Debiec J, Ledoux JE. 2004. Disruption of reconsolidation but not consolidation of auditory fear conditioning by noradrenergic blockade in the amygdala. Neuroscience 129: 267-272.

Debiec J, Bush DE, LeDoux JE. 2011. Noradrenergic enhancement of reconsolidation in the amygdala impairs extinction of conditioned fear in rats-a possible mechanism for the persistence of traumatic memories in PTSD. Depress Anxiety 28: 186-193.

Deutch AY, Goldstein M, Roth RH. 1986. Activation of the locus coeruleus induced by selective stimulation of the ventral tegmental area. Brain Res 363: $307-314$

Do-Monte FH, Allensworth M, Carobrez AP. 2010. Impairment of contextual conditioned fear extinction after microinjection of $\alpha$-1-adrenergic blocker prazosin into the medial prefrontal cortex. Behav Brain Res 211: 89-95.

Duvarci S, Pare D. 2014. Amygdala microcircuits controlling learned fear. Neuron 82: 966-980.

Egan TM, Henderson G, North RA, Williams JT. 1983. Noradrenaline-mediated synaptic inhibition in rat locus coeruleus neurones. J Physiol 345: 477-488.

Ennis M, Aston-Jones G. 1986. Evidence for self- and neighbor-mediated postactivation inhibition of locus coeruleus neurons. Brain Res 374: 299-305.

Fallon JH. 1981. Collateralization of monoamine neurons: mesotelencephalic dopamine projections to caudate, septum, and frontal cortex. J Neurosci 1: 1361-1368.
Fallon JH, Koziell DA, Moore RY. 1978. Catecholamine innervation of the basal forebrain. II. Amygdala, suprarhinal cortex and entorhinal cortex. J Comp Neurol 180: 509-532.

Feenstra MG, Vogel M, Botterblom MH, Joosten RN, de Bruin JP. 2001. Dopamine and noradrenaline efflux in the rat prefrontal cortex after classical aversive conditioning to an auditory cue. Eur J Neurosci 13: 1051-1054.

Fibiger HC, Mason ST. 1978. The effects of dorsal bundle injections of 6-hydroxydopamine on avoidance responding in rats. Br J Pharmacol 64: $601-605$

Fields HL, Margolis EB. 2015. Understanding opioid reward. Trends Neurosci 38: $217-225$

Fiorenza NG, Rosa J, Izquierdo I, Myskiw JC. 2012. Modulation of the extinction of two different fear-motivated tasks in three distinct brain areas. Behav Brain Res 232: 210-216.

Galvez R, Mesches MH, McGaugh JL. 1996. Norepinephrine release in the amygdala in response to footshock stimulation. Neurobiol Learn Mem 66: $253-257$

Harris GC, Fitzgerald RD. 1991. Locus coeruleus involvement in the learning of classically conditioned bradycardia. J Neurosci 11: 2314-2320.

Herry C, Johansen JP. 2014. Encoding of fear learning and memory in distributed neuronal circuits. Nat Neurosci 17: 1644-1654.

Herve-Minvielle A, Sara SJ. 1995. Rapid habituation of auditory responses of locus coeruleus cells in anaesthetized and awake rats. Neuroreport 6: $1363-1368$

Hugues S, Garcia R, Lena I. 2007. Time course of extracellular catecholamine and glutamate levels in the rat medial prefrontal cortex during and after extinction of conditioned fear. Synapse 61: 933-937.

Ishimatsu M, Williams JT. 1996. Synchronous activity in locus coeruleus results from dendritic interactions in pericoerulear regions. J Neurosci 16: $5196-5204$

Janak PH, Tye KM. 2015. From circuits to behaviour in the amygdala. Nature 517: 284-292.

Johansen JP, Cain CK, Ostroff LE, LeDoux JE. 2011. Molecular mechanisms of fear learning and memory. Cell 147: 509-524.

Johansen JP, Wolff SB, Luthi A, LeDoux JE. 2012. Controlling the elements: an optogenetic approach to understanding the neural circuits of fear. Biol Psychiatry 71: 1053-1060.

Johansen JP, Diaz-Mataix L, Hamanaka H, Ozawa T, Ycu E, Koivumaa J, Kumar A, Hou M, Deisseroth K, Boyden ES, et al. 2014. Hebbian and neuromodulatory mechanisms interact to trigger associative memory formation. Proc Natl Acad Sci 111: E5584-E5592.

Kalwani RM, Joshi S, Gold JI. 2014. Phasic activation of individual neurons in the locus ceruleus/subceruleus complex of monkeys reflects rewarded decisions to go but not stop. J Neurosci 34: 13656-13669.

Koob GF, Kelley AE, Mason ST. 1978. Locus coeruleus lesions: learning and extinction. Physiol Behav 20: 709-716.

LaLumiere RT, Niehoff KE, Kalivas PW. 2010. The infralimbic cortex regulates the consolidation of extinction after cocaine self-administration. Learn Mem 17: 168-175.

Lammel S, Lim BK, Malenka RC. 2014. Reward and aversion in a heterogeneous midbrain dopamine system. Neuropharmacology $76 \mathbf{P t}$ B: $351-359$.

Langlais PJ, Connor DJ, Thal L. 1993. Comparison of the effects of single and combined neurotoxic lesions of the nucleus basalis magnocellularis and dorsal noradrenergic bundle on learning and memory in the rat. Behav Brain Res 54: 81-90.

LeDoux JE. 2000. Emotion circuits in the brain. Annu Rev Neurosci 23: $155-184$.

Likhtik E, Stujenske JM, Topiwala MA, Harris AZ, Gordon JA. 2014. Prefrontal entrainment of amygdala activity signals safety in learned fear and innate anxiety. Nat Neurosci 17: 106-113.

Liu C, Placais PY, Yamagata N, Pfeiffer BD, Aso Y, Friedrich AB, Siwanowicz I, Rubin GM, Preat T, Tanimoto H. 2012. A subset of dopamine neurons signals reward for odour memory in Drosophila. Nature 488: 512-516.

Loughlin SE, Foote SL, Grzanna R. 1986. Efferent projections of nucleus locus coeruleus: morphologic subpopulations have different efferent targets. Neuroscience 18: 307-319.

Luo L, Callaway EM, Svoboda K. 2008. Genetic dissection of neural circuits. Neuron 57: 634-660.

Luppi PH, Aston-Jones G, Akaoka H, Chouvet G, Jouvet M. 1995. Afferent projections to the rat locus coeruleus demonstrated by retrograde and anterograde tracing with cholera-toxin B subunit and Phaseolus vulgaris leucoagglutinin. Neuroscience 65: 119-160.

Mason ST, Fibiger HC. 1979. Regional topography within noradrenergic locus coeruleus as revealed by retrograde transport of horseradish peroxidase. J Comp Neurol 187: 703-724.

Mason ST, Iversen SD. 1975. Learning in the absence of forebrain noradrenaline. Nature 258: 422-424. 
McGaugh JL. 2004. The amygdala modulates the consolidation of memories of emotionally arousing experiences. Annu Rev Neurosci 27: $1-28$.

McGaughy J, Ross RS, Eichenbaum H. 2008. Noradrenergic, but not cholinergic, deafferentation of prefrontal cortex impairs attentional set-shifting. Neuroscience 153: 63-71.

Mueller D, Porter JT, Quirk GJ. 2008. Noradrenergic signaling in infralimbic cortex increases cell excitability and strengthens memory for fear extinction. J Neurosci 28: 369-375.

Nader K, Hardt O. 2009. A single standard for memory: the case for reconsolidation. Nat Rev Neurosci 10: 224-234.

Nagai T, Satoh K, Imamoto K, Maeda T. 1981. Divergent projections of catecholamine neurons of the locus coeruleus as revealed by fluorescent retrograde double labeling technique. Neurosci Lett 23: $117-123$.

Nakamura S, Iwama K. 1975. Antidromic activation of the rat locus coeruleus neurons from hippocampus, cerebral and cerebellar cortices. Brain Res 99: 372-376.

Neophytou SI, Aspley S, Butler S, Beckett S, Marsden CA. 2001. Effects of lesioning noradrenergic neurones in the locus coeruleus on conditioned and unconditioned aversive behaviour in the rat. Prog Neuropsychopharmacol Biol Psychiatry 25: 1307-1321.

Newman LA, Darling J, McGaughy J. 2008. Atomoxetine reverses attentional deficits produced by noradrenergic deafferentation of medial prefrontal cortex. Psychopharmacology 200: 39-50.

Ornstein K, Milon H, McRae-Degueurce A, Alvarez C, Berger B, Wurzner HP. 1987. Biochemical and radioautographic evidence for dopaminergic afferents of the locus coeruleus originating in the ventral tegmental area. J Neural Transm 70: $183-191$.

Palkovits M, Saavedra JM, Jacoboqitz DM, Kizer JS, Zaborszky L, Brownstein MJ. 1977. Serotonergic innervation of the forebrain: effect of lesions on serotonin and tryptophan hydroxylase levels. Brain Res 130: $121-134$.

Paxinos G, Watson C. 1982. The rat brain in stereotaxic coordinates. Academic Press, New York.

Plaznik A, Kostowski W. 1980. Locus coeruleus lesions and avoidance behavior in rats. Acta Neurobiol Exp (Wars) 40: 217-225.

Quirarte GL, Galvez R, Roozendaal B, McGaugh JL. 1998. Norepinephrine release in the amygdala in response to footshock and opioid peptidergic drugs. Brain Res 808: $134-140$.

Rajkowski J, Majczynski H, Clayton E, Aston-Jones G. 2004. Activation of monkey locus coeruleus neurons varies with difficulty and performance in a target detection task. J Neurophysiol 92: 361-371.

Rasmussen K, Jacobs BL. 1986. Single unit activity of locus coeruleus neurons in the freely moving cat. II. Conditioning and pharmacologic studies. Brain Res 371: 335-344.

Reyes BA, Valentino RJ, Xu G, Van Bockstaele EJ. 2005. Hypothalamic projections to locus coeruleus neurons in rat brain. Eur J Neurosci 22: 93-106.

Room P, Postema F, Korf J. 1981. Divergent axon collaterals of rat locus coeruleus neurons: demonstration by a fluorescent double labeling technique. Brain Res 221: 219-230.

Sara SJ, Bouret S. 2012. Orienting and reorienting: the locus coeruleus mediates cognition through arousal. Neuron 76: 130-141.

Sara SJ, Segal M. 1991. Plasticity of sensory responses of locus coeruleus neurons in the behaving rat: implications for cognition. Prog Brain Res 88: $571-585$.

Schwarz LA, Miyamichi K, Gao XJ, Beier KT, Weissbourd B, DeLoach KE, Ren J, Ibanes S, Malenka RC, Kremer EJ, Luo L. 2015. Viral-genetic tracing of the input-output organization of a central noradrenaline circuit. Nature. doi: 10.1038/nature 14600

Sears RM, Fink AE, Wigestrand MB, Farb CR, de Lecea L, Ledoux JE. 2013. Orexin/hypocretin system modulates amygdala-dependent threat learning through the locus coeruleus. Proc Natl Acad Sci 110: 20260-20265.

Selden NR, Robbins TW, Everitt BJ. 1990. Enhanced behavioral conditioning to context and impaired behavioral and neuroendocrine responses to conditioned stimuli following ceruleocortical noradrenergic lesions: support for an attentional hypothesis of central noradrenergic function. J Neurosci 10: 531-539.

Senn V, Wolff SB, Herry C, Grenier F, Ehrlich I, Grundemann J, Fadok JP, Muller C, Letzkus JJ, Luthi A. 2014. Long-range connectivity defines behavioral specificity of amygdala neurons. Neuron 81: 428-437.

Simpson KL, Altman DW, Wang L, Kirifides ML, Lin RC, Waterhouse BD. 1997. Lateralization and functional organization of the locus coeruleus projection to the trigeminal somatosensory pathway in rat. J Comp Neurol 385: $135-147$.

Sotres-Bayon F, Quirk GJ. 2010. Prefrontal control of fear: more than just extinction. Curr Opin Neurobiol 20: 231-235.

Soya S, Shoji H, Hasegawa E, Hondo M, Miyakawa T, Yanagisawa M, Mieda M, Sakurai T. 2013. Orexin receptor-1 in the locus coeruleus plays an important role in cue-dependent fear memory consolidation. $J$ Neurosci 33: 14549-14557.

Swanson LW. 1982. The projections of the ventral tegmental area and adjacent regions: a combined fluorescent retrograde tracer and immunofluorescence study in the rat. Brain Res Bull 9: 321-353.

Tervo DG, Proskurin M, Manakov M, Kabra M, Vollmer A, Branson K, Karpova AY. 2014. Behavioral variability through stochastic choice and its gating by anterior cingulate cortex. Cell 159: 21-32.

Tovote P, Fadok JP, Luthi A. 2015. Neuronal circuits for fear and anxiety. Nat Rev Neurosci 16: 317-331.

Travagli RA, Dunwiddie TV, Williams JT. 1995. Opioid inhibition in locus coeruleus. J Neurophysiol 74: 518-528.

Tsaltas E, Gray JA, Fillenz M. 1984. Alleviation of response suppression to conditioned aversive stimuli by lesions of the dorsal noradrenergic bundle. Behav Brain Res 13: 115-127.

Tsaltas E, Schugens MM, Gray JA. 1989. Effects of lesions of the dorsal noradrenergic bundle on conditioned suppression to a CS and to a contextual background stimulus. Behav Brain Res 31: 243-256.

Tully K, Li Y, Tsvetkov E, Bolshakov VY. 2007. Norepinephrine enables the induction of associative long-term potentiation at thalamo-amygdala synapses. Proc Natl Acad Sci 104: 14146-14150.

Tye KM, Deisseroth K. 2012. Optogenetic investigation of neural circuits underlying brain disease in animal models. Nat Rev Neurosci 13: $251-266$.

Usher M, Cohen JD, Servan-Schreiber D, Rajkowski J, Aston-Jones G. 1999. The role of locus coeruleus in the regulation of cognitive performance. Science 283: 549-554.

Valentino RJ, Van Bockstaele E. 2015. Endogenous opioids: the downside of opposing stress. Neurobiol Stress 1: 23-32.

Van Bockstaele EJ, Colago EE, Aicher S. 1998a. Light and electron microscopic evidence for topographic and monosynaptic projections from neurons in the ventral medulla to noradrenergic dendrites in the rat locus coeruleus. Brain Res 784: 123-138.

Van Bockstaele EJ, Colago EE, Valentino RJ. 1998b. Amygdaloid corticotropin-releasing factor targets locus coeruleus dendrites: substrate for the co-ordination of emotional and cognitive limbs of the stress response. J Neuroendocrinol 10: 743-757.

Waddell S. 2013. Reinforcement signalling in Drosophila; dopamine does it all after all. Curr Opin Neurobiol 23: 324-329.

Waterhouse BD, Lin CS, Burne RA, Woodward DJ. 1983. The distribution of neocortical projection neurons in the locus coeruleus. J Comp Neurol 217: $418-431$.

Waterhouse BD, Border B, Wahl L, Mihailoff GA. 1993. Topographic organization of rat locus coeruleus and dorsal raphe nuclei: distribution of cells projecting to visual system structures. J Comp Neurol 336: $345-361$.

Yu AJ, Dayan P. 2005. Uncertainty, neuromodulation, and attention. Neuron 46: 681-692.

Received April 23, 2015; accepted in revised form June 22, 2015. 


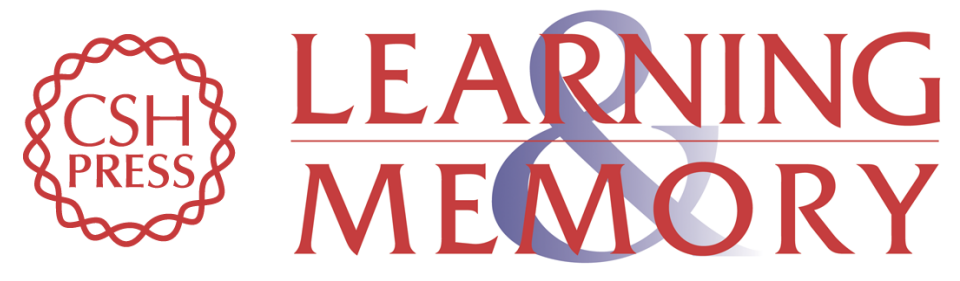

\section{Projection specificity in heterogeneous locus coeruleus cell populations: implications for learning and memory}

Akira Uematsu, Bao Zhen Tan and Joshua P. Johansen

Learn. Mem. 2015, 22:

Access the most recent version at doi:10.1101/lm.037283.114

References This article cites 106 articles, 21 of which can be accessed free at: http://learnmem.cshlp.org/content/22/9/444.full.html\#ref-list-1

Creative This article is distributed exclusively by Cold Spring Harbor Laboratory Press for the Commons License first 12 months after the full-issue publication date (see

http://learnmem.cshlp.org/site/misc/terms.xhtml). After 12 months, it is available under a Creative Commons License (Attribution-NonCommercial 4.0 International), as described at http://creativecommons.org/licenses/by-nc/4.0/.

Email Alerting Receive free email alerts when new articles cite this article - sign up in the box at the Service top right corner of the article or click here. 